\title{
Der administrative Aufwand der Ärzteschaft nimmt weiter zu
}

\author{
Bruno Trezzinia, Beatrix Meyer ${ }^{b}$, Melanie Ivankovic ${ }^{c}$, Cloé Jans $^{d}$, Lukas Goldere \\ a Dr. phil., wissenschaftlicher Mitarbeiter, Abteilung Stationäre Versorgung und Tarife, FMH; ${ }^{b}$ Leiterin Abteilung Stationäre Versorgung und Tarife, FMH; \\ ${ }^{c}$ Junior Projektleiterin gfs.bern; ${ }^{d}$ Leiterin operatives Geschäft gfs.bern; ${ }^{e}$ Co-Leiter gfs.bern
}

Der administrative Dokumentationsaufwand hat sich für die Spitalärzteschaft über die Jahre sukzessive erhöht. Dies könnte mit ein Grund dafür sein, dass die zwar nach wie vor hohe Arbeitszufriedenheit tendenziell abgenommen hat. Die Ärzteschaft zeigt sich grundsätzlich offen für Neuerungen wie z.B. Mindestfallzahlen. Der Blick auf das eigene Spital ist demgegenüber etwas skeptischer geworden.

1 Zur ausführlichen Studie vgl. Golder L, et al. (2019). Ärzteschaft offen für Veränderungen-Umgang der Spitäler mit Veränderungen aber nur für die Hälfte der Ärztinnen und Ärzte überzeugend. Schlussbericht (www.fmh $\mathrm{ch} \rightarrow$ Themen $\rightarrow$ Stationäre Tarife $\rightarrow$ Begleitforschung).
In persönlichen Gesprächen äussern sich Ärztinnen und Ärzte oftmals besorgt über den wachsenden administrativen Aufwand, der sie immer mehr von der eigentlichen medizinischen Arbeit abhält. In der medialen Öffentlichkeit wird hingegen eher die Art und Höhe der Entlohnung von Spitalärztinnen und -ärzten in den Vordergrund gerückt. Im Auftrag der FMH führt gfs.bern seit 2011 jährlich eine repräsentative Befra-

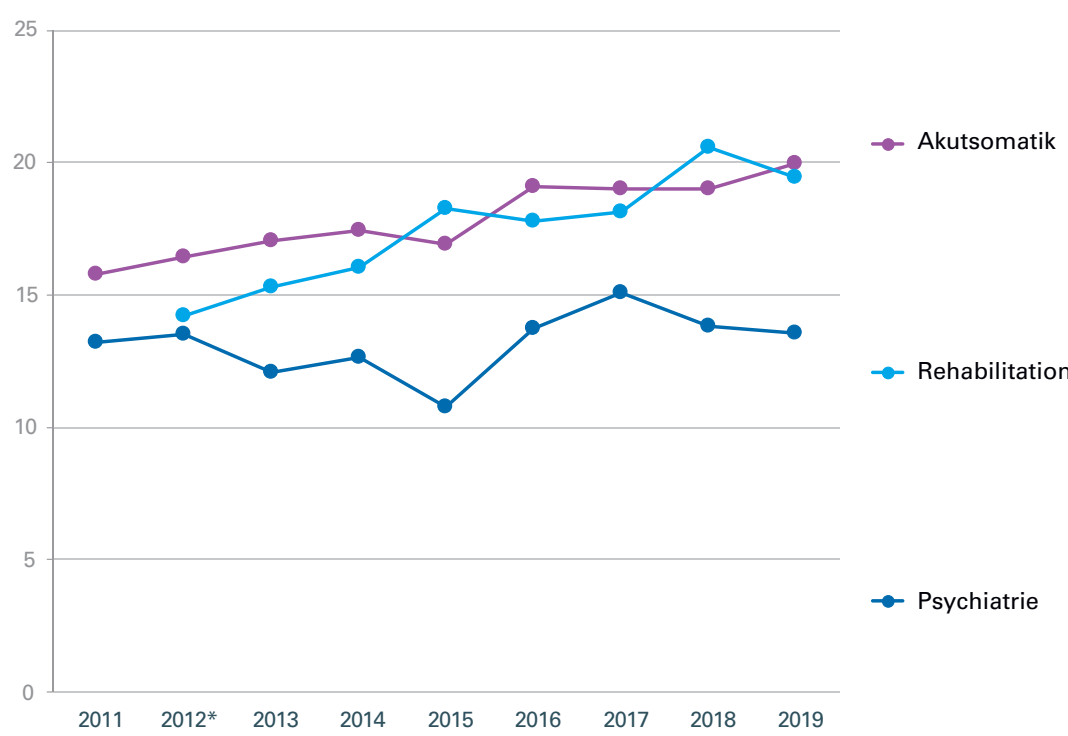

Abbildung 1: Trend im Vergleich des Zeitaufwands für «ärztliche Dokumentationsarbeit/ Patientendossier» (in \%-Mittelwerten).

(Frage: «Wenn Sie an den letzten normalen Arbeitstag denken, wie viel Zeit haben Sie dabei auf die folgenden Tätigkeiten angewendet? Bitte geben Sie Ihre Angaben in Minuten an.» N Akutsomatik jeweils ca. 940, N Psychiatrie jeweils ca. 130, N Rehabilitation jeweils ca. 70. * Rehabilitation 2011 und 2012 zusammen.) gung der Ärzteschaft durch, die solche und andere Themen einzuordnen hilft. ${ }^{1}$ An der aktuellsten Erhebung von Juni und Juli 2019 haben insgesamt 1572 Ärztinnen und Ärzte teilgenommen.

\section{Trend zeigt beim administrativen Aufwand seit Jahren nach oben}

Zwar machen medizinische patientennahe Tätigkeiten 2019 mit 34\% nach wie vor den grössten Anteil der Arbeitszeit der Ärztinnen und Ärzte in der Akutsomatik aus. Der tägliche Zeitaufwand für Dokumentationsarbeiten rund um das Patientendossier folgt aber mit einem Anteil von 20\% bereits auf dem zweiten Platz. Insbesondere in der Akutsomatik und in der Rehabilitation ist ein im Vergleich zur ersten Umfrage im Jahr 2011 besonders deutlicher Anstieg der für die Dokumentation aufgewendeten Zeit festzustellen (vgl. Abb. 1). Damals wendeten akutsomatisch tätige Ärztinnen und Ärzte dafür noch 86 Minuten pro Tag auf. Heute benötigen sie mit 119 Minuten täglich über eine halbe Stunde länger, um diese Aufgaben erledigen zu können. In der Rehabilitation sieht die Situation ähnlich aus: Wurden 2012 noch 82 Minuten benötigt, sind es heutzutage 118 Minuten pro Tag für die Dokumentation von Patientendaten. Im psychiatrischen Bereich stieg der Wert von 64 Minuten pro Tag im Jahr 2011 auf heute 76 Minuten. Bezogen auf die hierarchische Position der Befragten, ist der Zeitaufwand in der Akutsomatik bei den Assistenzärztinnen und -ärzten mit 167 Minuten pro Tag mit Abstand am höchsten; sie verbringen etwas mehr als $27 \%$ ihrer Arbeitszeit mit Dokumentation am Patientendossier 
und damit nahezu gleich viel Zeit wie für patientennahe Tätigkeiten.

\section{Hohe Arbeitszufriedenheit nimmt leicht ab}

Der steigende administrative Aufwand dürfte einer der Gründe sein, weshalb die Arbeitszufriedenheit der Ärztinnen und Ärzte über die vergangenen neun Jahre kontinuierlich gesunken ist. Nach wie vor sind jedoch rund 80\% der Ärztinnen und Ärzte in der Akutsomatik und in der Psychiatrie sowie knapp 70\% in der Rehabilitation sehr oder eher zufrieden mit ihrer Tätigkeit. Stabile über 90\% der Ärztinnen und Ärzte finden ihre Arbeit interessant und abwechslungsreich; mehrheitlich sind die Befragten auch sehr oder eher mit ihrem Lohn zufrieden. Bei einer deutlichen Mehrheit der Ärzteschaft herrscht jedoch ein konstant hoher Leistungs- und Zeitdruck. In der Akutsomatik geben knapp 80\% der Ärztinnen und Ärzte an, dass dies sehr oder eher zutrifft. Deutlich zugenommen hat zudem der Anteil von Ärztinnen und Ärzten, die meistens oder häufig unter Stress leiden; in der Akutsomatik stieg der Prozentsatz von 40\% im Jahr 2012 auf heute knapp 60\%.

\section{Ärzteschaft ist offen für Neuerungen und Reformen}

Die befragten Ärztinnen und Ärzte zeigen sich mehrheitlich offen für Veränderungen wie beispielsweise

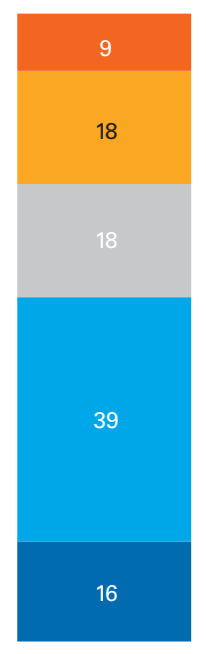

Mindestfallzahlen pro Spital

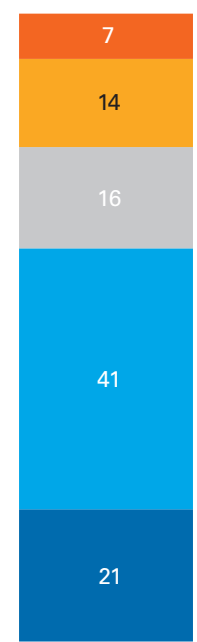

Mindestfallzahlen pro Operateur
Abbildung 2: Persönliche Meinung zu Mindestfallzahlen pro Spital und pro Operateur (nur Akutsomatik: in \%).

(Frage: «Was halten Sie persönlich von Mindestfallzahlen pro Spital / pro Operateur?», N = 1066.) die zunehmend angewendeten Mindestfallzahlen in kantonalen Leistungsaufträgen oder die Listen «ambulant vor stationär».

55\% der in der Akutsomatik tätigen Ärzteschaft finden Mindestfallzahlen pro Spital absolut oder eher gerechtfertigt (vgl. Abb. 2). Mit 62\% ist die Zustimmung im Falle von Mindestfallzahlen pro Operateur noch ausgeprägter. Jeweils etwas weniger als ein Fünftel der Befragten hat sich jedoch noch keine Meinung dazu gebildet.

\section{Mindestfallzahlen für Spitäler und für Opera-} teure werden von einer Mehrheit der Ärztinnen und Ärzte begrüsst.

Seit Anfang 2019 gilt zudem die Liste des Bundesamtes für Gesundheit zu «ambulant vor stationär». In der Liste werden sechs Gruppen von elektiven medizinischen Eingriffen definiert, die in der Regel ambulant durchgeführt werden müssen. 2019 ist in der Akutsomatik mit 53\% eine Mehrheit eher bis sehr mit der Einführung von «ambulant vor stationär»-Listen einverstanden, was einem Anstieg von 6 Prozentpunkten gegenüber dem Vorjahr entspricht. Eine gewisse Skepsis bezüglich der Ausweitung solcher Listen auf das eigene Fachgebiet ist jedoch teilweise weiterhin vorhanden, wobei sich diese im Vergleich zum Vorjahr reduzierte.

\section{Zunehmend kritischerer Blick auf das eigene Spital}

Zwar ist die Ärzteschaft offen für Veränderungen. Wie die Spitalverwaltung jedoch damit umgeht, wird zunehmend kritisch beurteilt. Nur noch knapp die Hälfte der befragten Ärzteschaft findet den Umgang mit Veränderungen, wie er von den Spitälern gepflegt wird, sehr oder eher gut. Eine solche Dynamik widerspiegelt sich auch im sinkenden Anteil von Ärztinnen und Ärzten, die der Ansicht sind, dass ihr Spital eine Strategie für die Positionierung im Wettbewerb hat. So sank bei der Akutsomatik der Ja-Anteil von einem Höchststand von 63\% im Jahr 2015 auf 48\% im Jahr 2019. Ferner gab zwar zwischen 2011 und 2015 noch ein steigender Anteil der akutsomatisch tätigen Ärztinnen und Ärzte an, dass die Zusammenarbeit mit der Spitalverwaltung konstruktiv verläuft. Seither zeigt sich jedoch eine abnehmende Tendenz, und der Anteil von «trifft sehr/ eher zu» lag 2019 nur noch bei rund 40\%. Eine zunehmend negativere Einschätzung zeigt sich darüber hinaus hinsichtlich der allgemeinen Arbeitsorganisation im Spital. Die Aussage, in ihrem Spital sei die allgemeine Arbeitsorganisation schlecht, wird in der Akutsomatik im Jahr 2019 von 39\% als sehr oder eher zutref- 


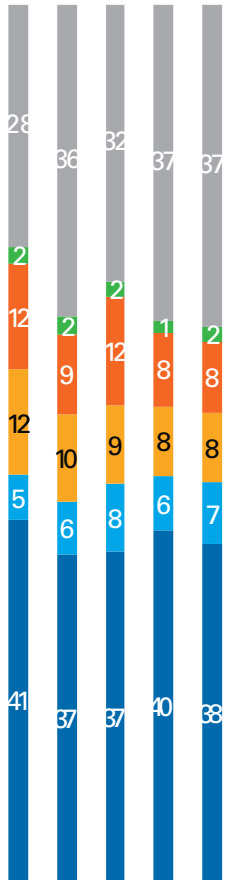

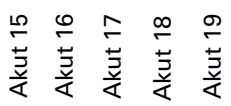
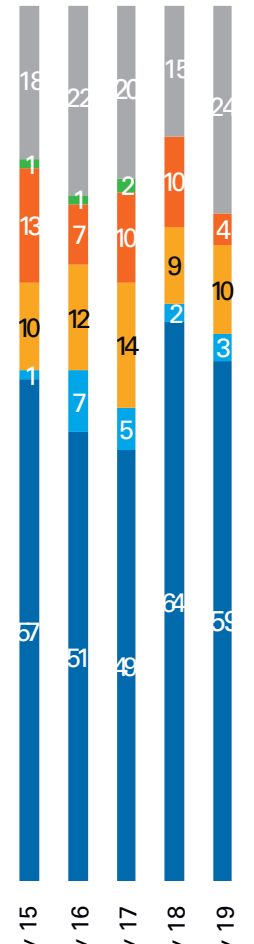

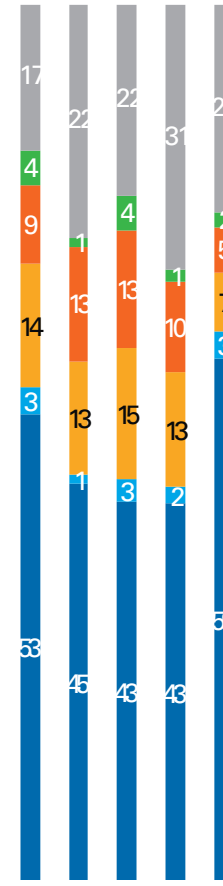

$\stackrel{\circ}{\circ} \approx \stackrel{\circ}{\circ}$ keine Angabe bei Fragen 1, 2 und 3

ausschliesslich andere

leistungsabhängige Lohnkomponenten

Einkünfte aus privatärztlicher (Honorar-) Tätigkeit (teilweise kombiniert mit anderen leistungsab. Lohnkomponenten)

- Bonuszahlungen (teilweise kombiniert mit anderen leistungsabhängigen Lohnkomponenten)

- keine persönliche leistungsabhängige Lohnkomponente

keine leistungsabhängige Lohnkomponente in Abteilung/Klinik

Abbildung 3: Trend im Vergleich der leistungsabhängigen Lohnkomponenten (in \%).

(Frage 1: «Existieren auf Ihrer Abteilung in Ihrer Klinik Entschädigungssysteme mit variablen, leistungsabhängigen Lohnkomponenten?», Frage 2: «Enthält Ihr persönliches Entschädigungssystem eine variable, leistungsabhängige Lohnkomponente?», Frage 3 (falls variable, leistungsabhängige Lohnkomponente): «Handelt es sich bei der variablen, leistungsabhängigen Lohnkomponente um: (a) Bonuszahlungen auf der Grundlage einer Zielvereinbarung (z.B. Erreichen von quantitativen oder qualitativen Zielen), (b) Einkünfte aus privatärztlicher Tätigkeit/Honorartätigkeit am Spital, (c) andere, welche?, (d) keine Angaben.» Fälle, die sowohl Bonuszahlungen als auch Honorartätigkeiten angegeben haben, wurden der Gruppe «Bonuszahlungen» zugeordnet. N Akutsomatik jeweils ca. 940, N Psychiatrie jeweils ca. 130, N Rehabilitation jeweils ca. 70.)

fend angesehen; 2011 waren es erst 35\%. Schliesslich sind über die letzten fünf Jahre betrachtet kontinuierlich weniger Ärztinnen und Ärzte stolz auf ihr Spital; heute sind es noch $74 \%$.

\section{Bonuszahlungen verlieren an Bedeutung}

Zielbezogene Boni können Fehlanreize setzen, insbesondere wenn die Ziele an Mengenvorgaben geknüpft sind. Deshalb empfiehlt die FMH seit längerem, in Spitalarztverträgen auf zielbezogene Bonusvereinbarungen zu verzichten. ${ }^{2}$ Der Anteil der Ärztinnen und Ärzte, die Boni erhalten, hat in der Akutsomatik denn auch im Vergleich zu 2015 von 12 auf 8\% und in der Rehabilitation von 14 auf 7\% abgenommen, während er in der Psychiatrie mit 10\% gleichgeblieben ist (vgl. Abb. 3). Zudem ist bei den Bonusbezügern in der Akutsomatik der Anteil der Boni am Gesamtlohn weiter gesunken und beträgt gegenwärtig durchschnittlich noch etwas über $15 \%$. Betrachtet nach Hierarchiestufe erhalten in der Akutsomatik 7\% der Oberärztinnen und -ärzte, 17\% der leitenden Ärztinnen und Ärzte und 16\% der Chefärztinnen und -ärzte zielbezogene Boni.

\section{Mehr Zeit für die Patientinnen und Patienten schaffen}

Genügend Zeit für die Patientinnen und Patienten zu haben ist nicht nur für das Patientenwohl relevant, sondern auch für die Zufriedenheit der Ärztinnen und Ärzte. Damit diese nicht weiter abnimmt, gilt es insbesondere, den administrativen Aufwand zu reduzieren und die Arbeitsorganisation in den Spitälern zu verbessern. Dies nicht zuletzt, um für die besten Talente und Nachwuchskräfte attraktiv zu bleiben. ${ }^{3}$ Für die Positionierung der Spitäler im Wettbewerb ist es zentral, dass sich die Ärztinnen und Ärzte primär auf ihre Patientinnen und Patienten konzentrieren können. Schliesslich kann der Spitalverwaltung kaum etwas Besseres geschehen, als dass ihre Ärztinnen und Ärzte stolz auf ihr Spital sind und dies in ihrem Umfeld kundtun.

Bildnachweis

gfs.bern 